EPJ Web of Conferences 49, 17012 (2013)

DOI: $10.1051 /$ epjconf/20134917012

(C) Owned by the authors, published by EDP Sciences, 2013

\title{
Single top-quark production measurements with ATLAS at the LHC
}

\author{
A. Lleres ${ }^{1, a}$ on behalf of the ATLAS collaboration \\ ${ }^{1}$ Laboratoire de Physique Subatomique et de Cosmologie, Université Joseph Fourier and CNRS/IN2P3 and Institut National \\ Polytechnique de Grenoble, Grenoble, France
}

\begin{abstract}
We present cross-section measurements of single top-quark production in the $t$-channel and associated $W t$ production modes using $p p$ collision data recorded with the ATLAS detector at the LHC. The $t$-channel total cross-section is measured at $\sqrt{s}=7 \mathrm{TeV}$ and at $\sqrt{s}=8 \mathrm{TeV}$ with an integrated luminosity of $1.04 \mathrm{fb}^{-1}$ and $5.8 \mathrm{fb}^{-1}$, respectively. The $t$-channel top-quark and top-antiquark production cross sections and their ratio are also measured at $7 \mathrm{TeV}$ with $4.7 \mathrm{fb}^{-1}$ of data. Evidence for the $W t$ associated production is found at $7 \mathrm{TeV}$ in the dileptonic channel using a luminosity of $2.05 \mathrm{fb}^{-1}$. The measured cross-sections as well as the top-antitop ratio are in good agreement with the Standard Model predictions. The coupling strength $\left|V_{t b}\right|$ is determined from the ratio of the measured to the theoretically predicted cross sections assuming that $\left|V_{t s}\right|$ and $\left|V_{t d}\right|$ are small. The extracted values are all compatible with the Standard Model and with previous measurements.
\end{abstract}

\section{Introduction}

At hadron colliders top-quarks are predominantly produced in pairs (top-antitop) via the strong interaction. Alternative production modes proceed via the weak interaction involving a $W t b$ vertex, leading to a single top-quark intermediate state. Three subprocesses contribute to single top-quark production: the exchange of a virtual $W$ boson in the $t$-channel or in the $s$-channel and the associated production of a top-quark and an on-shell $W$ boson. The single top-quark final state provides a direct probe of the $W t b$ coupling and is sensitive to many models of new physics. The measurement of the production cross section constrains the absolute value of the quark-mixing (CKM) matrix element $V_{t b}$ without assumptions about the number of quark generations.

The observation of single top-quark production was first reported at the Tevatron by both $\mathrm{D} \varnothing$ [1] and CDF [2] experiments. The observations are consistent with the Standard Model (SM) expectation for the $t$-channel and $s$ channel processes. The third single top-quark production mechanism $(W t)$ has an expected cross-section too small at the Tevatron to be observed.

At the Large Hadron Collider (LHC), first measurements of single top-quark production in the $t$-channel and $W t$ modes have been recently reported by the ATLAS [3, 4] and CMS [5] collaborations at a centre-of-mass (CM) energy of $7 \mathrm{TeV}$. They also show good agreement with the SM expectation which is at approximate next-tonext-leading order (NNLO) $\sigma_{t}=64.6_{-2.0}^{+2.7} \mathrm{pb}$ for the leading $t$-channel process and $\sigma_{W t}=15.7 \pm 1.1 \mathrm{pb}$ for the (sub-leading) $W t$ production mode. These values are calculated for a top-quark mass of $172.5 \mathrm{GeV}$ and the uncer-

\footnotetext{
a e-mail: 1leres@1psc.in2p3.fr
}

tainties come from the renormalisation and factorisation scales and from the parton distribution functions (PDFs). At the energy of $8 \mathrm{TeV}$, a cross section $\sigma_{t}=87.8_{-1.9}^{+3.4} \mathrm{pb}$ is calculated at NNLO for the dominant $t$-channel process.

In this contribution, we present the results obtained for the $t$-channel and $W t$ single top-quark production using the LHC $p p$ collisions data recorded at $\sqrt{s}=7$ and $\sqrt{s}=8 \mathrm{TeV}$ with the ATLAS detector. The cross-sections are extracted and values of the magnitude of the CKM matrix element $\left|V_{t b}\right|$ are derived. Complete lists of references for these results can be found in the original publications $[3,4,6,7]$.

\section{Data and simulation samples}

The analyses reported here use the LHC proton-proton collision data at centre-of-mass energies of 7 and $8 \mathrm{TeV}$ collected in 2011 and 2012 with the ATLAS detector [8]. The selected events were recorded based on single electron and muon triggers. For the $t$-channel measurements, the analyzed data samples correspond to integrated luminosities of $1.04 \pm 0.04 \mathrm{fb}^{-1}$ (total cross section) and $4.71 \pm 0.18 \mathrm{fb}^{-1}$ (top and antitop cross sections) at $7 \mathrm{TeV}$ and $5.8 \pm 0.2 \mathrm{fb}^{-1}$ at $8 \mathrm{TeV}$. The $W t$ analysis is based on a data sample recorded at $7 \mathrm{TeV}$ and corresponding to an integrated luminosity of $2.05 \pm 0.08 \mathrm{fb}^{-1}$.

Samples of Monte Carlo (MC) simulated events for all three single top-quark processes and for the top-quark pair process $(t \bar{t})$ are produced using dedicated generators (leading-order (LO) AcERMC and next-to-leading order (NLO) MC@NLO) and normalized using NNLO crosssections. The background contribution from Gauge boson $(W / Z)$ production in association with jets is simulated using the LO ALPGEN generator and the diboson processes $(W W, W Z, Z Z)$ using the ALPGEN or HERWIG

This is an Open Access article distributed under the terms of the Creative Commons Attribution License 2.0, which permits unrestricted use, distribution, and reproduction in any medium, provided the original work is properly cited. 


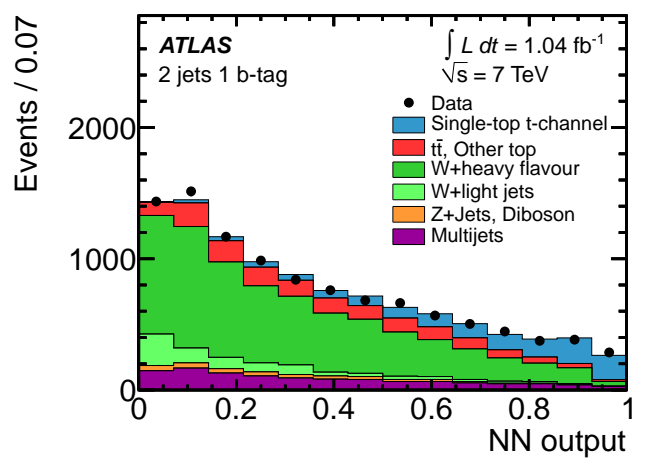

Figure 1. Neural network output distribution for the 2-jet samples at $7 \mathrm{TeV}$ [4]. All component distributions are normalised to the likelihood fit result except the multijet normalisation which is derived from the fit to the $E_{\mathrm{T}}^{\text {miss }}$ distribution.

generators. The hadronization is performed by PYTHIA or with HERWIG in connection with the JIMMY underlying event model. After event generation, all samples are passed through the full simulation of the ATLAS detector based on GEANT4 and are reconstructed using the same procedure as collision data. More details on the used simulation samples are given in $[3,4]$.

\section{Object definition}

Electron candidates are reconstructed using a clusterbased algorithm and are required to have a transverse energy $E_{\mathrm{T}}>25 \mathrm{GeV}$ and a pseudorapidity for the calorimeter cluster $\left|\eta_{\mathrm{cc}}\right|<2.47$. Events with electrons falling in the calorimeter barrel-endcap transition region $\left(1.37<\left|\eta_{\mathrm{cl}}\right|<\right.$ $1.52)$ are rejected. High-quality electron candidates are selected using a set of cuts which include stringent requirements on the matching between the track and the calorimeter cluster. Isolation criteria are in addition required by applying cuts on the calorimeter transverse energy of all cells and on the transverse momentum of all tracks within cones of radius $\Delta R=0.2$ or 0.3 around the electron direction.

Muon candidates are reconstructed by combining track segments found in the inner detector and in the muon spectrometer and are required to have a transverse momentum $p_{\mathrm{T}}>25 \mathrm{GeV}$ and $|\eta|<2.5$. Selected muons must additonally satisfy a series of cuts on the number of track hits present in the various tracking sub-detectors. Muon candidates are required to be isolated using equivalent criteria as applied to electron candidates.

Hadronic jets are reconstructed from calorimeter clusters using the anti- $k_{t}[9]$ algorithm with a radius parameter of 0.4. The response of the calorimeter is corrected by $p_{\mathrm{T}^{-}}$and $\eta$-dependent factors which are applied to each jet to provide an average energy scale correction. Jets are required to have $E_{\mathrm{T}}>25 \mathrm{GeV}$ (at $7 \mathrm{TeV}$ ) or $30 \mathrm{GeV}(8 \mathrm{TeV}$ ) and $|\eta|<2.5$ (Wt) or 4.5 (t-channel). Jets overlapping with selected electron candidates within $\Delta R<0.2$ are removed. For the $t$-channel analyses, jets originating from bottom quarks are tagged in the region $|\eta|<2.5$ by reconstructing secondary and tertiary vertices from the tracks associated

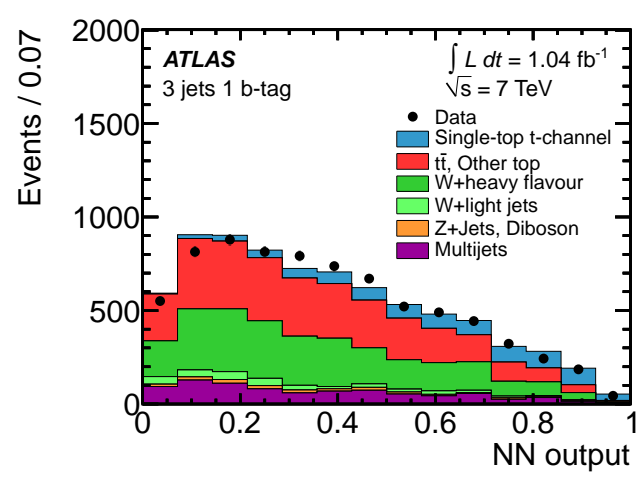

Figure 2. Same as Figure 1 for the 3-jet samples [4].

with each jet and combining lifetime-related information with a neural network. A threshold is applied to the $b$ tagging algorithm output corresponding to a $b$-tagging efficiency of about $50-60 \%$ and a light-quark jet rejection factor of about 500. The missing transverse energy $\left(E_{\mathrm{T}}^{\text {miss }}\right)$ is calculated using clusters of adjacent cells and corrected for the presence of electrons, muons and jets.

\section{Event selection}

For the $t$-channel measurements, the event selection requires exactly one electron or muon candidate, exactly two or three jets and $E_{\mathrm{T}}^{\text {miss }}>25 \mathrm{GeV}(7 \mathrm{TeV})$ or $30 \mathrm{GeV}$ $(8 \mathrm{TeV})$. A trigger matching requirement is applied where the lepton must lie within $\Delta R<0.15$ of its trigger-level object. Contribution from multijet background is additionally reduced through a requirement on the transverse mass of the lepton- $E_{\mathrm{T}}^{\text {miss }}$ system: $m_{\mathrm{T}}(W)>\left(60 \mathrm{GeV}-E_{\mathrm{T}}^{\text {miss }}\right)$ or $m_{\mathrm{T}}(W)>30 / 50 \mathrm{GeV}$ depending on the luminosity and on the CM energy. The final signal sample is obtained by requiring in addition that exactly one jet is $b$-tagged.

For the $W t$ analysis, events with exactly two charged leptons (electron or muon) with opposite signs are selected and classified in the $e e, \mu \mu$ and $e \mu$ categories. Only events with at least one jet without any $b$-tagging requirement are considered and in addition the missing transverse momentum of the event is required to be greater than $50 \mathrm{GeV}$. In the $e e$ and $\mu \mu$ channels, the invariant mass of the lepton pair $\left(m_{\ell \ell}\right)$ is required to satisfy $m_{\ell \ell}<81 \mathrm{GeV}$ or $m_{\ell \ell}>101 \mathrm{GeV}$ in order to reduce the contamination from $Z$ boson decays. Furthermore, in all channels, the $Z$ to $\tau \tau$ background is reduced by applying a cut on the sum of the two angles in the transverse plane between each lepton and the missing transverse momentum direction.

\section{Background estimation}

For single top final states, the main background contributions originate from top pair production, QCD-multijet events (events with a fake lepton from jet misidentification) and from $W$ boson production in association with jets (this process contributes mainly as background of the $t$ channel events). Smaller background contributions come 


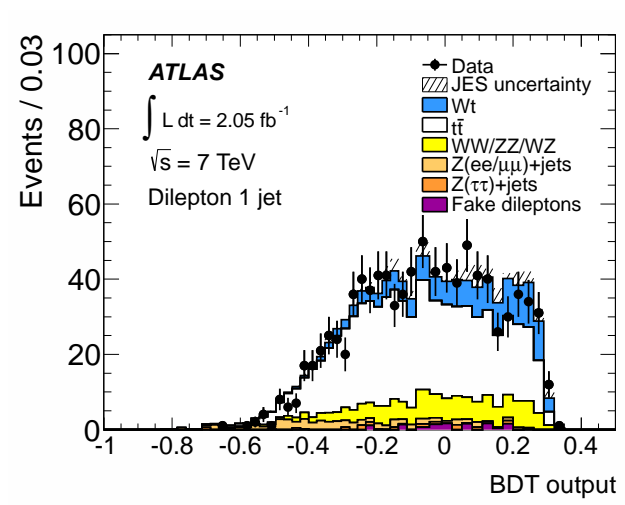

Figure 3. BDT output distribution for the 1-jet samples at $7 \mathrm{TeV}$ [3]. The $W t$ signal is normalised to the theory prediction.

from $Z+$ jets and diboson production as well as from the other single top processes. These smaller backgrounds and the $t \bar{t}$ contribution are normalised to their NLO or NNLO theoretical predictions. For the $W+$ jets background, the kinematic distributions are taken from samples of simulated events while the normalisation of the flavour composition is derived from data.

For the $t$-channel measurements, the multijet background normalisation is obtained through a binned maximum-likelihood fit to the $E_{\mathrm{T}}^{\text {miss }}$ distribution in the data using a data-derived template for the multijet background and templates from Monte Carlo simulation for all other processes. The multijet template is created using collision events triggered by a single low- $p_{\mathrm{T}}$ jet. These events are then selected by replacing the electron requirement by a jet requirement.

In the Wt analysis, the multijet background contamination is normalised using the so-called matrix method which relates the observed sample composition in terms of selected leptons of different qualities to its true composition in terms of real and fake leptons.

\section{Signal and background discrimination}

To separate $t$-channel or $W t$ signal events from background, several kinematic variables are combined into one discriminant by employing a multivariate analysis based on a neural network (NN) ( $t$-channel) or on the boosted decision trees technique (BDT) $(W t)$, both exploiting correlations between the variables. The highest-ranking variables are chosen for the training of the NN and BDT classifiers.

The fifteen highest-ranking variables defined from an optimisation procedure are used as inputs to the $t$-channel NN. For the 2-jet selection, the most discriminant observable is the invariant mass of the $b$-tagged jet, the charged lepton and the neutrino, which is an estimator of the topquark mass for signal events (the transverse momentum of the neutrino is given by the $x$ - and $y$-components of the $E_{\mathrm{T}}^{\text {miss }}$ vector while the unmeasured $z$-component is inferred by imposing a $W$ boson mass constraint on the leptonneutrino system). For the 3 -jet selection, the two most dis-

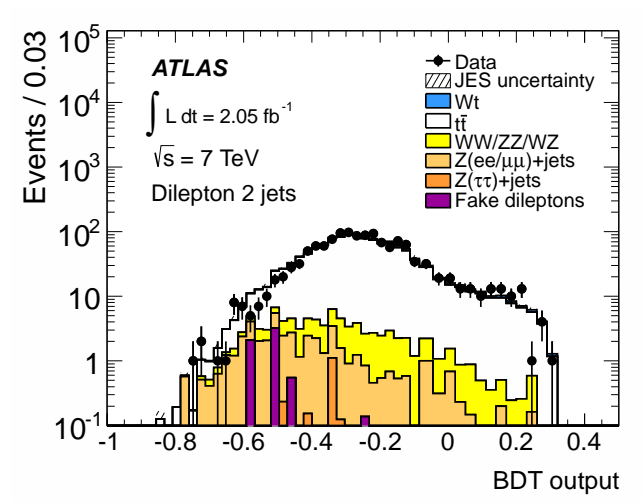

Figure 4. Same as Figure 3 for the 2-jet samples [3].

criminant variables are the invariant mass of the two leading jets and the absolute value of the difference in pseudorapidity of the leading and lowest $p_{\mathrm{T}}$ jets. For $W t$ discrimination, twenty-two variables with significant separation power are used as input to the BDT. The most powerful variable is the magnitude of the vectorial sum of the $p_{\mathrm{T}}$ of the leading jet, leptons and missing transverse momentum.

The NN output distributions obtained at $7 \mathrm{TeV}$ are shown in Figures 1 and 2 for events with two and three jets, respectively. Figures 3 and 4 display the BDT output distributions for selected $W t$ events with one and two jets, respectively. For both channels, a good agreement is obtained between the distributions for data and MC simulation.

\section{Cross-section and $\left|V_{t b}\right|$ measurements}

To extract the signal cross-section, a maximum-likelihood fit is performed to the complete multivariate output distributions for the datasets corresponding to the different jet multiplicities: the 2-jet and 3-jet samples for the $t$-channel measurements and the 1-jet, 2 -jet and $\geq 3$-jet events for the $W t$ observation are simultaneously fitted.

Systematic uncertainties on the normalisation of the individual backgrounds and on the signal acceptance as well as uncertainties on the shape of each individual prediction affect the measured cross-sections. The impact of the systematic uncertainties is estimated using a frequentist method based on the generation of a large number of correlated pseudo-experiments. The distribution of the extracted cross-section is an estimator of the probability density function of all possible outcomes of the measurement and it is used to estimate the uncertainty on the actual measurement. Different sources of uncertainties are taken into account: object modeling (residual differences between data and MC simulations for the reconstruction and energy calibration of jets, electrons and muons), Monte Carlo generators (modeling of the single top signal and $t \bar{t}$ background, of the parton showers and hadronisation, and of the amount of initial-state and final-state radiation (ISR/FSR)), parton distribution functions (PDFs), theoretical cross-section normalisation, background normalisation to data (QCD-multijet and $W+$ jets) and luminosity. 


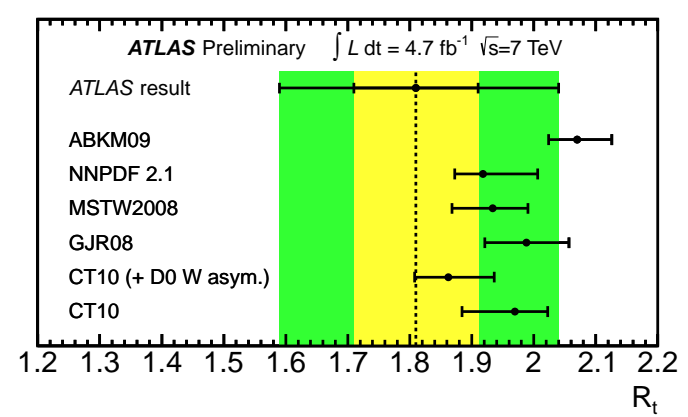

Figure 5. $R_{t}$ values calculated at $\sqrt{s}=7 \mathrm{TeV}$ for different NLO PDFs [6]. The error contains the uncertainty on the renormalisation and factorisation scales. The black line indicates the central value of the measured $R_{t}$ and the combined statistical and systematic uncertainty is shown in green (the yellow error band represents the statistical uncertainty).

The NN-based analysis of the $t$-channel events yields the following total cross sections:

$$
\sigma_{t}=83 \pm 4(\text { stat })_{-19}^{+20} \text { (syst) pb at } \sqrt{s}=7 \mathrm{TeV} \text {, }
$$$$
\sigma_{t}=95 \pm 2 \text { (stat) } \pm 18 \text { (syst) pb at } \sqrt{s}=8 \mathrm{TeV} \text {. }
$$

At $7 \mathrm{TeV}$, the main sources of systematic uncertainties come from ISR/FSR (14\%), the $b$-tagging modeling (13\%) and jet energy scale (6\%). At $8 \mathrm{TeV}$, the ISR/FSR and $b$ tagging uncertainties have a reduced impact on the measurement (8-9\%).

The cross-sections of the top-quark and top-antiquark production in the $t$-channel and their ratio $R_{t}$ measured at $7 \mathrm{TeV}$ are:

$$
\begin{aligned}
& \sigma_{t}(t)=53.2 \pm 1.7(\text { stat }) \pm 10.6(\text { syst }) \mathrm{pb} \\
& \sigma_{t}(\bar{t})=29.5 \pm 1.54(\text { stat }) \pm 7.3(\text { syst }) \mathrm{pb} \\
& R_{t}=\sigma_{t}(t) / \sigma_{t}(\bar{t})=1.81 \pm 0.10(\text { stat })_{-0.20}^{+0.21}(\text { syst })
\end{aligned}
$$

Adding the measurement of the top-quark and antitopquark cross-sections results in a total cross-section $\sigma_{t}=$ $82.7 \pm 2.3$ (stat) \pm 17.9 (syst) pb which is in excellent agreement with the direct measurement based on $1.04 \mathrm{fb}^{-1}$ of data (value given in the previous paragraph). The main sources of systematic uncertainty for $R_{t}$ come from the background normalisation (5\%), ISR/FSR (4\%) and the jet energy scale (4\%). The measured value of $R_{t}$ is compared to the predictions obtained with different PDF sets in Figure 5. It is in agreement with the predictions that vary between 1.86 and 2.07 depending upon the choice for the $u$-quark and the $d$-quark PDFs.

For the $W t$ cross-section measurement at $7 \mathrm{TeV}$, the result of the fit of the BDT output distribution is:

$$
\sigma_{W t}=16.8 \pm 2.9 \text { (stat) } \pm 4.9 \text { (syst) } \mathrm{pb} \text {. }
$$

This result is found to be incompatible with the background-only hypothesis at the $3.3 \sigma$ level, the expected sensitivity assuming the Standard Model production rate being $3.4 \sigma$.

A direct determination of the CKM matrix element $\left|V_{t b}\right|$ can be done from the measured single top crosssections assuming that $\left|V_{t b}\right| \gg\left|V_{t s}\right|,\left|V_{t d}\right|$ ( $\sigma$ is proportional to $\left|V_{t b}\right|^{2}$ ). In the Standard Model $\left|V_{t b}\right|$ is close to one. The value of $\left|V_{t b}\right|^{2}$ is extracted by dividing the observed single top cross-section by the SM expectation and the experimental and theoretical uncertainties are added in quadrature. The results obtained for $\left|V_{t b}\right|$ are the following: $\left|V_{t b}\right|=1.13_{-0.13}^{+0.14}$ from the $t$-channel cross-section measured at $7 \mathrm{TeV},\left|V_{t b}\right|=1.04_{-0.11}^{+0.10}$ from the measurement at $8 \mathrm{TeV}$ and $\left|V_{t b}\right|=1.03_{-0.19}^{+0.16}$ from the $W t$ cross-section measured at $7 \mathrm{TeV}$. These three results are compatible between them and with the direct measurements performed at the Tevatron $[10,11]$.

\section{Conclusions}

This contribution summarizes measurements of the crosssection of single top-quark production in the $t$-channel with the ATLAS detector in $p p$ collisions at $\sqrt{s}=7$ and $8 \mathrm{TeV}$. Evidence for the production of single top-quark events in the $W t$ production mode in the dileptonic decay channel is also reported. The measurements are based on neural network and boosted decision tree discrimination to separate signal events from background contaminations. All extracted cross-section values, plotted in Figure 6, are in good agreement with the Standard Model predictions. A direct determination of the CKM matrix element $\left|V_{t b}\right|$ is performed from the observed cross-sections and the values are compatible with the Standard Model and with previous measurements from the different colliders and experiments.

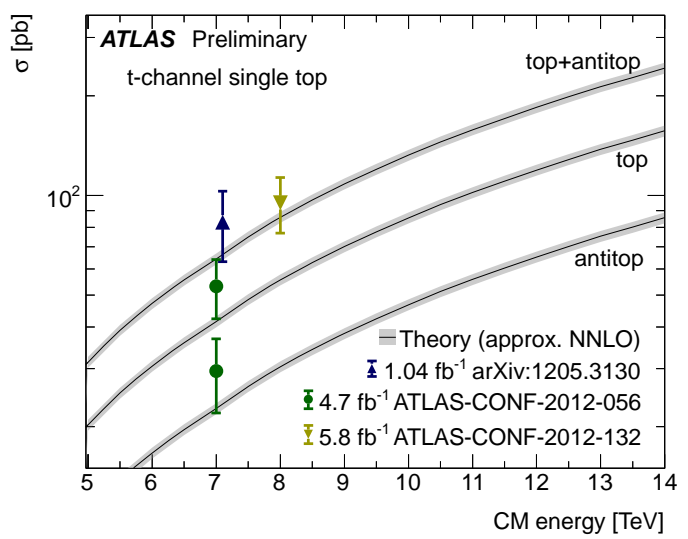

Figure 6. Summary of the ATLAS $\sqrt{s}=7 \mathrm{TeV}$ and $\sqrt{s}=8 \mathrm{TeV}$ single top cross-section measurements [7] with comparison to the approximate NNLO calculated values.

\section{References}

[1] CDF Collaboration, Phys. Rev. Lett. 103, 092002 (2009) and Phys. Rev. D 82, 112005 (2010).

[2] DØ Collaboration, Phys. Rev. Lett. 103, 092001 (2009) and Phys. Lett. B 705, 313 (2011).

[3] ATLAS Collaboration, Phys. Lett. B 716, 142 (2012).

[4] ATLAS Collaboration, Phys. Lett. B 717, 330 (2012).

[5] CMS Collaboration, Phys. Rev. Lett. 107, 091802 (2011) and arXiv:1209.4533 [hep-ex].

[6] ATLAS Collaboration, ATLAS-CONF-2012-056, http://cds.cern.ch/record/1453783. 
[7] ATLAS Collaboration, ATLAS-CONF-2012-132, http://cds.cern.ch/record/1478371.

[8] ATLAS Collaboration, JINST 3, S08003 (2008).

[9] M. Cacciari, G.P. Salam, G. Soyez, JHEP 080463 (2008).
[10] CDF Collaboration, CDF/PUB/TOP/PUBLIC/10793 (2012).

[11] DØ Collaboration, Phys. Rev. D 84, 112001 (2011). 